\title{
ORDERED COMMUTATIVE SEMIGROUPS OF THE SECOND KIND ${ }^{1}$
}

\section{A. H. CLIFFORD}

By an ordered commutative semigroup of the first kind (abbreviated "o.c.s.I") we mean a system $S(0,<)$ consisting of a set $S$ endowed with a binary operation $o$ and a binary relation $<$ such that the following axioms are satisfied.

I. $S$ is a commutative semigroup with respect to $\circ$, i.e. the associative law, $a \circ(b \circ c)=(a \circ b) \circ c$, and the commutative law, $a \circ b=b \circ a$, hold $(a, b, c$ in $S)$.

II. $S$ is totally (=linearly=simply) ordered by $<$.

III. If $a$ and $b$ are elements of $S$ such that $a<b$, then $a \circ c \leqq b \circ c$ for all $c$ in $S$.

Although this notion is very extensive, it does not include the ordered multiplicative system of all real numbers, in which multiplication by a negative number inverts the direction of any inequality. Let $S(\mathrm{o},<)$ satisfy just I and II above. An element $c$ of $S$ is called a conserver if $a<b(a, b$ in $S)$ implies $c \circ a \leqq c \circ b$, and an inverter if $a<b$ implies $c \circ a \geqq c \circ b$. $S(\circ,<)$ will be called an ordered commutative semigroup of the second kind (abbreviated "o.c.s. II") if it satisfies I, II, and IV below.

IV. Every element of $S$ is either a conserver or an inverter, or both.

Systems of this nature (in fact more general systems) have been investigated by Dov Tamari $[2 ; 3]$. His definition of conserver (inverter) is more restrictive: $a<b$ implies $c \circ a<c \circ b(c \circ a>c \circ b)$. If $c$ is a conserver or inverter in his sense, $c$ is cancellable $(c \circ a=c \circ b$ implies $a=b$ ). I have taken the liberty of relaxing the definition so as to apply to noncancellable elements as well; the term "strict conserver (inverter)" may be used for his concept.

The main objective of the first part of the present paper is to show that the set $P$ of conservers of $S$ and the set $Q$ of inverters of $S$ are convex. (A subset $A$ of $S$ is convex if $a \in A, a^{\prime} \in A$, and $a<x<a^{\prime}$ imply $x \in A$.)

An ultimate objective is to construct all o.c.s.II's from o.c.s.I's. In the second part of the paper we give such a construction for a fairly restricted class of such semigroups. This was suggested by recent work of Haskell Cohen and L. I. Wade [1].

Received by the Editors February 3, 1958.

1 This paper was prepared with the partial support of the National Science Foundation grant to Tulane University. 
Notation. We shall write $a b$ instead of $a \circ b$. If $A$ and $B$ are subsets of $S$, then (1) $A B$ denotes the set of all $a b$ with $a$ in $A, b$ in $B$; (2) $A<B$ means $a<b$ for all $a$ in $A, b$ in $B$; (3) $A \backslash B$ means the set of elements of $A$ not in $B$.

1. Location of conservers and inverters. Throughout this section, $S$ will denote an o.c.s.II. $P$ and $Q$ will denote the sets of conservers and inverters, respectively, of $S$. The elements of $N=P \cap Q$ will be called null elements. Evidently $P^{2} \subseteq P, Q^{2} \subseteq P, P Q \subseteq Q$. In particular, $P$ is a subsemigroup of $S$, and is an o.c.s.I. $N$ is an ideal of $S$, i.e. $S N \subseteq N$, if it is not empty.

If $S$ contains a zero element 0 , i.e. $a 0=0 a=0$ for all $a$ in $S$, we shall say that an element $a$ of $S$ is positive if $a>0$ and negative if $a<0$. We say that $a$ and $b$ have the same sign if they are both positive or both negative, and have opposite sign if one is positive and the other negative.

LemMA 1. Let $S$ have a zero element 0 . Let $a$ and $b$ be elements of $S$. If $a$ and $b$ are both conservers or both inverters, but have opposite sign, then $a b=0$. If $a$ is a conserver and $b$ an inverter, or vice-versa, and they have the same sign, then $a b=0$.

Proof. Suppose $a<0<b$. If $a$ and $b$ are both conservers, $a<0$ implies $a b \leqq 0 b=0$, and $0<b$ implies $0=a 0 \leqq a b$, whence $a b=0$. If $a$ and $b$ are both inverters, $a<0$ implies $a b \geqq 0 b=0$, and $0<b$ implies $0=a 0 \geqq a b$, and again we conclude $a b=0$.

Suppose $a$ is a conserver and $b$ an inverter, and $a<0, b<0 . a<0$ implies $a b \geqq 0$ since $b$ is an inverter. $b<0$ implies $a b \leqq 0$ since $a$ is a conserver. Hence $a b=0$. The case $a>0, b>0$ is similar.

An element $a$ of $S$ is a null if and only if $a b=a c$ for all $b, c$ in $S$. For if, say, $b<c$, then $a b \leqq a c$ because $a$ is a conserver, and $a b \geqq a c$ because $a$ is an inverter. From this we see that $a$ is a null if and only if $a b=a^{2}$ for all $b$ in $S$, i.e. $a S=\left\{a^{2}\right\}$.

LEMMA 2. $S$ contains a null element if and only if $S$ contains a zero element 0 . An element $a$ of $S$ is a null if and only if $a S=\{0\}$. The set $N$ of null elements of $S$ is a convex ideal containing 0.

Proof. Let $a$ be a null element of $S$. Then $a^{2}$ is a zero element of $S$. For if $b$ is any element of $S, a^{2} b=a(a b)=a^{2}$. Conversely, if $S$ contains a zero element 0 , then 0 is evidently a null element. Since the zero element is unique, we have shown that $a^{2}=0$ for any null $a$. Thus $a S=\left\{a^{2}\right\}=\{0\}$ for any null $a$, and conversely if $a S=\{0\}$ then $a$ is clearly null. 
We have noted above that $N$ is an ideal. To show that it is convex, let $a \in N, b \in N, c \in S$, and $a<c<b$. Let $x \in S$. Then $0=a x \leqq c x \leqq b x=0$ if $x$ is a conserver, and $0=a x \geqq c x \geqq b x=0$ if $x$ is an inverter. In either event, $c x=0$. Hence $c S=\{0\}$ and $c \in N$.

REMARK 1 . The above proof of the convexity of $N$ carries over to the following: the set of annihilators of any element or subset of $S$ is convex.

Lemma 3. If $N, P \backslash N$, and $Q \backslash N$ are all nonempty, then $P \backslash N<N$ $<Q \backslash N$ or $Q \backslash N<N<P \backslash N$.

Proof. Assume first that $S$ contains arbitrarily large conservers. Then $S$ cannot contain a positive non-null inverter. For suppose $a$ is an inverter, and $a>0$. We proceed to show that $a$ is null.

Let $x>0$. By hypothesis there exists a conserver $b \geqq x . a b=0$ by Lemma 1 , and hence $a x=0$, from $0<x \leqq b$ and Remark 1 . Let $x<0$. If $x$ is an inverter, then $a x=0$ by Lemma 1 . If $x$ is a conserver, let $c$ be a conserver $\geqq a ; c$ exists, by assumption. Then $c x=0$ by Lemma 1 , and hence $a x=0$ from $0<a \leqq c$ and Remark 1 .

Similarly we show: if $S$ contains arbitrarily large inverters, then $S$ cannot contain a positive non-null conserver; if $S$ contains arbitrarily small conservers (inverters), then $S$ cannot contain a negative nonnull inverter (conserver). From these the lemma follows at once.

Lemma 4. If $S$ does not contain a zero element, then $P<Q$ or $Q<P$.

Proof. Since $S=P \cup Q$ and $P \cap Q$ is empty, by Lemma 2 , it suffices to show that $P$ and $Q$ are convex.

Suppose, by way of contradiction, that $a<b<c$ with $a, c$ in $P$ and $b$ in $Q$. Since $b$ is an inverter, $a<b<c$ implies $a b \geqq b^{2} \geqq b c$. Since $a$ is a conserver, $b<c$ implies $a b \leqq a c$. Since $c$ is a conserver, $a<b$ implies $a c \leqq b c$. Hence $b c \leqq b^{2} \leqq a b \leqq a c \leqq b c$, whence $b c=b^{2}$. But $b c \in Q P \subseteq Q$, $b^{2} \in Q^{2} \subseteq P$, contrary to the fact that $P \cap Q$ is empty. The proof of the convexity of $Q$ is similar.

We combine the foregoing lemmas into the following theorem.

THEOREM 1. Let $S$ be an ordered commutative semigroup of the second kind. Let $P$ be the set of conservers, $Q$ the set of inverters of $S$, and $N=P \cap Q$ the set of nulls of $S$. If $N$ is empty, then $P<Q$ or $Q<P$. If $N$ is not empty, then $S$ has a zero element $0, a \in N$ if and only if aS $=\{0\}$, and $N$ is a convex ideal of $S$. If $P \backslash N$ and $Q \backslash N$ are not empty then $N$ lies between them; in particular, the sets $P, Q, N, P \backslash N$ and $Q \backslash N$ are all convex.

REMARK 2. If $Q \backslash N$ is empty then $S$ is of the first kind. $P \backslash N$ need 
not be convex in this case. In fact we may take any two o.c.s.I's, $S_{1}$ and $S_{2}$, containing zero elements $0_{1}$ and $0_{2}$, respectively, with $0_{1}$ the greatest element of $S_{1}$ and $0_{2}$ the least element of $S_{2}$, and let $S$ be their union, with $0_{1}$ and $0_{2}$ identified ( $=0$, say). Order $S$ so as to preserve the given order in $S_{1}$ and $S_{2}$ as already given, and make $S_{1}<S_{2} \backslash\{0\}$. Define products within $S_{1}$ and $S_{2}$ as already given, and for $a_{1} \in S_{1}, a_{2} \in S_{2}$, define $a_{1} a_{2}=a_{2} a_{1}=0 . S$ becomes thereby an o.c.s.I. From Lemma 1 it follows that any o.c.s.I with interior zero element can be obtained in this fashion from its subsemigroups $S_{1}$ of all nonnegative elements and $S_{2}$ of all non-positive elements.

Remark 3. If $P \backslash N$ is empty, $Q \backslash N$ need not be convex. For example, let $S=\left\{a_{1}<a_{2}<c<0<d<b_{1}<b_{2}\right\}$ with all products =0 except $a_{i} a_{j}=d$ and $b_{i} b_{j}=c($ all $i, j=1,2)$. We note that if $P \backslash N$ is empty, then $S^{2} \subseteq N$, and so $S^{3}=\{0\}$. For $S=Q$, whence $S^{2}=Q^{2} \subseteq P=P \cap Q=N$.

2. Construction of a certain class of o.c.s.II's. Let $S$ and $S^{\prime}$ be o.c.s.II's. By a homomorphism of $S$ into $S^{\prime}$ we mean a mapping $f$ of $S$ into $S^{\prime}$ satisfying (i) $f(a b)=f(a) f(b)$ for all $a, b$ in $S$, and (ii) if $a<b$ then $f(a) \leqq f(b)$. By a congruence relation $\rho$ in $S$ we mean an equivalence relation such that $a \rho b$ implies $a c \rho b c$ for all $c$ in $S . \rho$ is called convex if each congruence class is convex. Let $[a]$ denote the congruence class to which $a$ belongs ( $a$ in $S$ ). The factor semigroup $S / \rho$ consists of all the classes $[a]$, with product defined by $[a] \cdot[b]$ $=[a b]$. If $\rho$ is convex, we can order $S / \rho$ by defining $[a]<[b]$ if $[a] \neq[b]$ and $a<b . S / \rho$ is then an o.c.s.II, and the canonical mapping $a \rightarrow[a]$ is a homomorphism of $S$ onto $S / \rho$. If $f$ is a homomorphism of $S$ onto $S^{\prime}$, and we define $a \rho b$ if and only if $f(a)=f(b)$, then $\rho$ is a convex congruence relation in $S$, and $[a] \rightarrow f(a)$ is an isomorphism of $S / \rho$ onto $S^{\prime}$.

An example of a convex congruence relation which we shall meet later is $\tau(k)$, where $k$ is a fixed element of $S$, defined as follows: $a \tau(k) b$ if and only if $k a=k b$.

We proceed now to investigate the class of o.c.s.II's $S$ satisfying the following conditions:

(a) $S$ is not of the first kind.

(b) $S$ contains a zero element 0 , not an endpoint.

(c) $S$ has endpoints $\delta<u$, and $u$ is the identity element of $S$ (i.e. $u a=a u=a$, all $a$ in $S$ ).

(d) $L \subseteq \delta R$, where $L=[\delta, 0]$ and $R=[0, u]$.

By (a), $S$ contains a non-null inverter. $S$ also contains the non-null conserver $u$. Since $S$ contains an identity element, $N$ must consist of 0 alone. By Theorem 1, 0 lies between the set $P \backslash N$ of non-null conservers and the set $Q \backslash N$ of non-null inverters. Since $u \in P \backslash N$, it 
follows that $R=P$ and $L=Q$. In particular, $\delta \in Q$, and so $\delta R \subseteq Q P \subseteq Q$ $=L$. (d) requires that $\delta R$ be all of $L$.

The above notation is that of Cohen and Wade [1]. They are concerned with the case where $S$ is a real interval, $x \circ y$ is a continuous function of the two variables $x$ and $y$, and product $o$ is not necessarily commutative. (d) holds because the continuous mapping $x \rightarrow \delta x$ maps the connected set $R$ onto the connected set $\delta R$ containing 0 and $\delta$, hence containing $L$. There are two main cases in [1], depending on whether $L^{2} \subseteq L$ or $L^{2} \subseteq R$. We are concerned only with the latter. Theorem 2 below may be regarded as an algebraic extract from Cohen and Wade's determination of $S$ in this case. The present treatment will be self-contained.

$R$ is a subsemigroup of $S$, and is an o.c.s.I, with the zero element at the lower end and the identity element at the upper end. We take the point of view that $R$ is known, and our objective is to construct $S$ in all possible ways from $R$.

First assume that $S$ is given. Then $k=\delta^{2} \in R$. Let $\phi(x)=\delta x(x \in R)$. By (d), $\phi$ maps $R$ onto $L$. Multiplication in $S$ is completely determined by that in $R$, and by $k$ and $\phi$, as follows $(x, y \in R)$ :

$$
x \phi(y)=\phi(x) y=\phi(x y), \quad \phi(x) \phi(y)=k x y .
$$

Moreover the order relation in $L$ is determined by that in $R$, and by $\phi$, as follows $(x, y \in R)$ :

$$
\phi(x)<\phi(y) \text { if and only if } \phi(x) \neq \phi(y) \text { and } x>y .
$$

We define the relation $\rho$ in $R$ by $x \rho y$ if and only if $\phi(x)=\phi(y) . \rho$ is evidently a convex congruence relation in $R$, and $L$ is order-antiisomorphic with $R / \rho$. (There is no question here of product-isomorphism, since $L$ is not closed under multiplication in $S$.) We note moreover that $\rho \leqq \tau(k)$, i.e. $x \rho y$ implies $x \tau(k) y$. For $x \rho y$ means $\delta x=\delta y$, whence $k x=\delta^{2} x=\delta^{2} y=k y$.

Now let us travel the same road in the opposite direction, starting with an o.c.s.I, $R$, with endpoints $0<u, 0$ the zero element and $u$ the identity element of $R$. Let $\rho$ be a convex congruence relation in $R$, and let $k$ be an element of $R$ such that $\rho \leqq \tau(k)$. Let $L$ be the set of congruence classes of $R \bmod \rho$. Let $\phi$ be the canonical mapping of $R$ onto $L$, so that $x \rho y$ if and only if $\phi(x)=\phi(y)$. Define an order relation in $L$ by (2). (Thus $L$ is an ordered set order-anti-isomorphic with $R / \rho$.)

We now identify 0 and $\phi(0)$, and let $S=L \cup R$, ordering $S$ so as to preserve the order already defined in $L$ and $R$, and so that $L<R \backslash\{0\}$. $S$ has the least element $\delta=\phi(u)$. Define product in $S$ by (1). To see that the definition is single-valued, let $\phi(x)=\phi\left(x^{\prime}\right)$ and $\phi(y)=\phi\left(y^{\prime}\right)$, 
with $x, y, x^{\prime}, y^{\prime}$ in $R$. Then $x \phi(y)=\phi(x y)$ and $x \phi\left(y^{\prime}\right)=\phi\left(x y^{\prime}\right)$. But from $y \rho y^{\prime}$ we have $x y \rho x y^{\prime}$, i.e. $\phi(x y)=\phi\left(x y^{\prime}\right)$. Likewise, $\phi(x) \phi(y)=k x y$ and $\phi\left(x^{\prime}\right) \phi\left(y^{\prime}\right)=k x^{\prime} y^{\prime}$. But from $x \rho x^{\prime}$ and $y \rho y^{\prime}$ we have $x y \rho x^{\prime} y^{\prime}$, and hence $k x y=k x^{\prime} y^{\prime}$ from $\rho \leqq \tau(k)$.

Verification of associativity is routine; for example:

$$
\begin{aligned}
\phi(x) y \cdot \phi(z) & =\phi(x y) \cdot \phi(z)=k(x y) \cdot z, \\
\phi(x) \cdot y \phi(z) & =\phi(x) \cdot \phi(y z)=k x \cdot(y z) ; \\
\phi(x) \phi(y) \cdot \phi(z) & =k x y \cdot \phi(z)=\phi((k x y) x), \\
\phi(x) \cdot \phi(y) \phi(z) & =\phi(x) \cdot k y z=\phi(x(k y z)) .
\end{aligned}
$$

We must show that every element of $R$ is a conserver and every element of $L$ and inverter. Here we shall verify the latter only; the former is similar.

Let $b \in L$, and let $s<t(s, t$ in $S)$. We are to show $b s \geqq b t$. Since $\phi$ maps $R$ onto $L, b=\phi(a)$ with $a$ in $R$. If $s \in R$ then $t \in R$ also, and $b s=\phi(a) s=\phi(a s), b t=\phi(a) t=\phi(a t)$. From $s<t$ and $a, s, t \in R$ we have $a s \leqq a t$, whence $\phi(a s) \geqq \phi(a t)$ by (2), i.e. $b s \geqq b t$. If $s \in L$ and $t \in R$, then $b s \in R$ and $b t \in L$, whence $b s \geqq b t$. If $s \in L$ and $t \in L$, then $s=\phi(x)$, $t=\phi(y)$ for some $x, y$ in $R$. From $s<t$ and (2) we have $x>y$. Now $b s=\phi(a) \phi(x)=k a x, b t=\phi(a) \phi(y)=k a y$. From $x>y$ and $k, a, x, y \in R$ we have $k a x \geqq k a y$, i.e. $b s \geqq b t$.

We summarize the foregoing in the following theorem.

THEOREM 2. Let $R$ be an ordered commutative semigroup (of the first kind) with endpoints $0<u, 0$ being the zero element and $u$ the identity element of $R$. Let $\rho$ be a convex congruence relation in $R$, and $k$ an element of $R$, such that xpy implies $k x=k y$. Let $L$ be the set of congruence classes of $R \bmod \rho$, and let $\phi$ be the canonical mapping of $R$ onto $L$. Order $L$ by (2). Let $S=L \cup R$ with 0 and $\phi(0)$ identified, and order $S$ so that $L<R \backslash\{0\}$. Define product in $S$ by (1). Then $S$ is an ordered commutative semigroup of the second kind with properties (a)-(d). Conversely, every ordered commutative semigroup of the second kind with properties (a)-(d) is obtainable from $R=[0, u]$ by the above construction.

\section{REFERENCES}

1. Haskell Cohen and L. I. Wade, Clans with zero on an interval, Trans. Amer. Math. Soc. vol. 88 (1958) pp. 523-534.

2. Dov Tamari, Groupoides reliés et demi-groupes ordonnés, C. R. Acad. Sci. Paris vol. 228 (1949) pp. 1184-1186.

3. - Groupoüdes ordonnés. L'ordre lexicographique pondéré, C. R. Acad. Sci. Paris, vol. 228 (1949) pp. 1909-1911.

Tulane UNIVERsity of Louisiana 